\title{
Assessment of liver fibrosis with liver and spleen magnetic resonance elastography, serum markers in chronic liver disease
}

\author{
Xiao-Pei Wang ${ }^{1}$, Yu Wang ${ }^{2}$, Hong Ma ${ }^{2}$, Han Wang ${ }^{2}$, Da-Wei Yang ${ }^{1}$, Xin-Yan Zhao ${ }^{2}$, Er-Hu Jin ${ }^{1}$, \\ Zheng-Han Yang ${ }^{1}$ \\ ${ }^{1}$ Department of Radiology, ${ }^{2}$ Liver Research Center, Beijing Friendship Hospital, Capital Medical University, Beijing 100050, China
}

Correspondence to: Er-Hu Jin, MD; Zheng-Han Yang, MD. No.95 Yongan Road, Xicheng District, Beijing 100050, China. Email: erhujin@263.net; cjr.yangzhenghan@vip.163.com.

Background: The accurate assessment of liver fibrosis is essential for patients with chronic liver disease. A liver biopsy is an invasive procedure that has many potential defects and complications. Therefore, noninvasive assessment techniques are of considerable value for clinical diagnosis. Liver and spleen magnetic resonance elastography (MRE) and serum markers have been proposed for quantitative and noninvasive assessment of liver fibrosis. This study aims to compare the diagnostic performance of liver and spleen stiffness measured by MRE, fibrosis index based on the 4 factors (FIB-4), aspartate aminotransferase-toplatelet ratio index (APRI), and their combined models for staging hepatic fibrosis.

Methods: One hundred and twenty patients with chronic liver disease underwent MRE scans. Liver and spleen stiffness were measured by the MRE stiffness maps. Serum markers were collected to calculate FIB4 and APRI. Liver biopsies were used to identify pathologic grading. Spearman's rank correlation analysis evaluated the correlation between the parameters and fibrosis stages. Receiver operating characteristic (ROC) analysis evaluated the performance of the four individual parameters, a liver and spleen stiffness combined model, and an all-parameters combined model in assessing liver fibrosis.

Results: Liver stiffness, spleen stiffness, FIB-4, and APRI were all correlated with fibrosis stage $(r=0.87$, $0.64,0.65$, and 0.51 , respectively, all $\mathrm{P}<0.001)$. Among the 4 individual diagnostic markers, liver stiffness showed the highest values in staging $\mathrm{F} 1-4, \mathrm{~F} 2-4, \mathrm{~F} 3-4$ and $\mathrm{F} 4$ (AUC $=0.89,0.97,0.95$, and 0.95 , all $\mathrm{P}<0.001)$. The AUCs of the liver and spleen stiffness combined model in the F1-4, F2-4, F3-4, and F4 staging groups were 0.89, 0.97, 0.95, and 0.96, respectively (all $\mathrm{P}<0.001$ ). The corresponding AUCs of the all-parameters combined model were 0.90, 0.97, 0.95, and 0.96 (all $\mathrm{P}<0.001$ ). The AUCs of the liver and spleen stiffness combined model were significantly higher than those of APRI, FIB-4 in the F2-4, F3-4, and $\mathrm{F} 4$ staging groups (all $\mathrm{P}<0.05$ ). Both combined models were not significantly different from liver stiffness in staging liver fibrosis (all $\mathrm{P}>0.05$ ).

Conclusions: Liver stiffness measured with MRE had better diagnostic performance than spleen stiffness, APRI, and FIB-4 for fibrosis staging. The combined models did not significantly improve the diagnostic value compared with liver stiffness in staging fibrosis.

Keywords: Magnetic resonance elastography (MRE); fibrosis index based on the 4 factors (FIB-4); aspartate aminotransferase-to-platelet ratio index (APRI); liver stiffness; spleen stiffness

Submitted Oct 15, 2019. Accepted for publication Apr 28, 2020.

doi: 10.21037/qims-19-849

View this article at: http://dx.doi.org/10.21037/qims-19-849 


\section{Introduction}

The prevalence, morbidity, and mortality rates of chronic liver disease have increased worldwide $(1,2)$. The common pathological processes for chronic liver disease include liver inflammation, liver fibrosis, liver cirrhosis, hepatocellular carcinoma, liver failure, and other adverse outcomes. Liver fibrosis is a pivotal stage and is closely associated with a poor prognosis (3). Many studies have confirmed that fibrogenesis accompanied by the deposition of extracellular matrix such as collagen, laminin, elastin, and fibronectin is dynamic and reversible (4). Therefore, the assessment of liver fibrosis plays a key role in clinical management. Liver biopsy and pathological grading are good references for diagnosing liver fibrosis. However, this method of examination is invasive and has many drawbacks and complications, such as sampling errors, hemorrhaging, infection, prohibitive cost, and a low patients' acceptance rate $(5,6)$. Hence, noninvasive assessment techniques hold considerable value for the clinical diagnosis, staging, and monitoring of liver fibrosis.

Two major noninvasive methods for liver fibrosis staging are serum markers and imaging techniques. Serum fibrosis markers such as fibrosis index based on the 4 factors (FIB-4) (7), and aspartate aminotransferase-to-platelet count ratio index (AST-to-platelet ratio index, APRI) (8) can be easily obtained from routine laboratory tests. Currently, a single marker or a combination of these markers can achieve satisfactory effects in clinical application. However, some indexes are not specific to the liver and may be influenced by other, extrahepatic diseases. These indexes are considered useful for identifying advanced fibrosis or cirrhosis; however, staging is difficult, and the determination of cutoff values for each hepatic fibrosis stage is controversial $(9,10)$.

Imaging methods, especially elastography, have been a hot research topic in recent decades. Magnetic resonance elastography (MRE) can be used to assess the viscoelastic properties of the liver and has high repeatability and excellent diagnostic performance for hepatic fibrosis (11-13). MRE can overcome some limitations of ultrasound elastography, such as obesity, operator dependence, and ascites, which are contraindicated only in FibroScan (10). Also, some magnetic resonance (MR)-based techniques, such as intravoxel incoherent motion (IVIM), diffusion kurtosis imaging (DKI), T1 $\rho$, dynamic contrast-enhanced (DCE), and perfusion, have been used in staging liver fibrosis. Besides, new post-processing approaches can accurately stage early fibrosis; for instance, Wang et al. demonstrated that it was possible to analyze IVIM without $\mathrm{b}=0$ images (14), and $\mathrm{Xu}$ et al., showed that it was possible to apply texture analysis (15). Many studies have indicated that MRE has higher accuracy and reliability than other MR-based techniques for the detection and stratification of liver fibrosis (16-18). Nevertheless, liver stiffness measured by MRE may be influenced by confounding factors, such as inflammation and portal hypertension. Most papers have focused on severe fibrosis, leading to a lack of consensus regarding the specific thresholds for different stages of fibrosis $(19,20)$.

Congestive splenomegaly is a common feature in patients with cirrhosis. As chronic liver disease progresses, the hemodynamics and morphologic characteristics of the spleen change accordingly. Spleen stiffness measured by MRE is positively correlated with liver stiffness and increases with liver fibrosis aggravation $(21,22)$. Some studies have reported that measurement of spleen stiffness is promising for noninvasive evaluation of the complications arising from liver-related diseases (e.g., portal hypertension and esophageal varices) $(23,24)$. However, there are insufficient studies for evaluating the diagnostic efficiency of spleen stiffness for the assessment of liver fibrosis. Moreover, clinicians need guidelines for the application of various available noninvasive markers (e.g., MRE and serum markers) for patients with distinct types of liver disease.

This study aims to compare the diagnostic performance of liver and spleen stiffness measured by MRE, common serum markers (FIB-4, APRI), imaging data combined model (liver and spleen stiffness), imaging data and serum markers combined model (liver and spleen stiffness, APRI, FIB-4) for staging hepatic fibrosis.

\section{Methods}

\section{Patients}

This study was approved by our institutional review board. Written informed consent was obtained from all patients.

This was a retrospective study of patients with clinically diagnosed chronic liver disease who underwent MRE (182 patients, from March 2018 through January 2019). The exclusion criteria were: (I) younger than 18 years old (excluded patients: 0); (II) pregnant and lactating women (excluded patients: 0); (III) technical failure of MRE due to iron disposition in the liver (excluded patients: 4); (IV) technical failure of MRE due to motion artifacts (excluded 


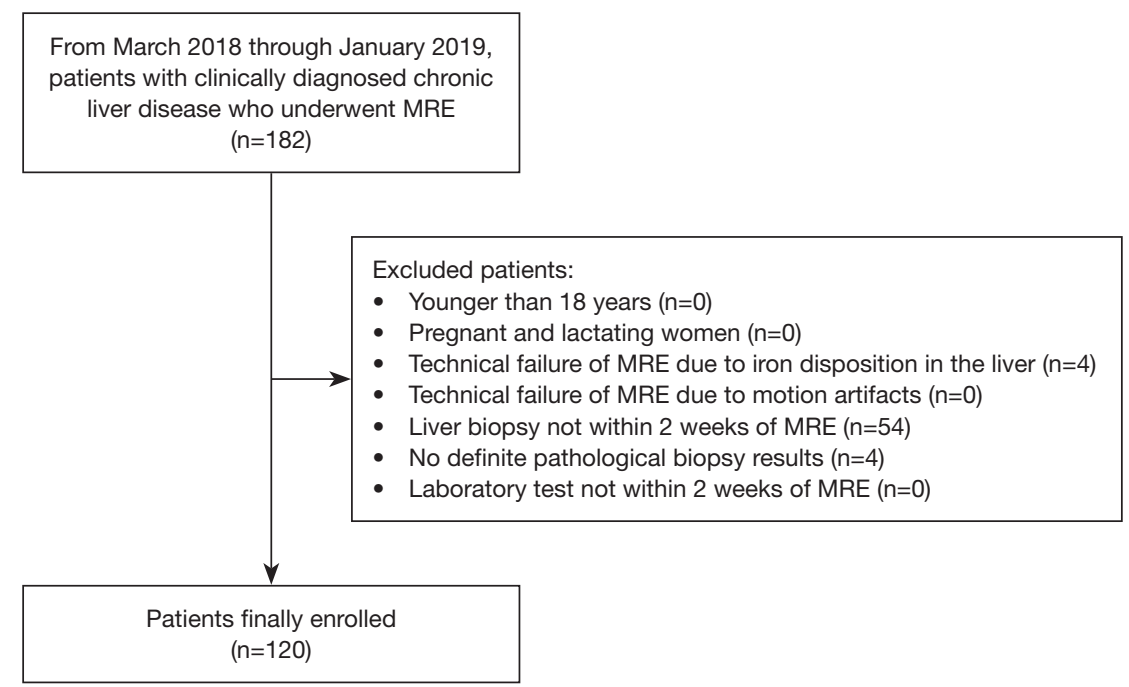

Figure 1 Flowchart of the study population. MRE, magnetic resonance elastography.

patients: 0); (V) liver biopsy was not performed within 2 weeks of MRE (excluded patients: 54); (VI) no definite pathological biopsy results (excluded patients: 4); and (VII) laboratory test was not performed within 2 weeks of MRE (excluded patients: 0). The total number of excluded patients was 62. Figure 1 shows the flowchart of our study.

All patients underwent a liver biopsy. A liver pathologist with 20 years of experience, who was blinded to the patients' clinical information, read and diagnosed the liver fibrosis stage and inflammation activity according to the METAVIR (25) and Scheuer score (26) systems. Fibrosis was staged on a $0-4$ scale: F0, no fibrosis; F1, portal fibrosis without septa, mild fibrosis; F2, portal fibrosis with a few septa, significant fibrosis; F3, numerous septa without cirrhosis, advanced fibrosis; F4, cirrhosis. The inflammatory activity was graded on a $0-4$ scale (G0-4).

\section{Magnetic resonance imaging (MRI)}

Patients were asked to fast for at least 6 hours before the scan. MRI was performed on a $3.0 \mathrm{~T}$ MRI scanner (Discovery 750W, GE, Milwaukee, USA) with a 16-channel phased-array coil. Multislice MRE was performed using the breath-hold 2D spin echo-echo planar imaging (SE-EPI) sequence before the administration of the contrast agent. An active audio driver (Resoundant Inc., USA), located outside the scan room, could generate acoustic pressure waves. A 19-cm-diameter passive driver was connected to the active audio driver via a flexible tube that was positioned over the right upper abdomen of supine patients at the level of the xiphoid process (27). An elastic tape was used to secure the passive driver to the body tightly, and the vibrations entered the patients. We tried to balance the acquisition time and scan range and then set the sequence parameters, which are summarized in Table 1.

\section{Analysis of liver and spleen stiffness}

The post-processing software "Volume Viewer" (version 13.0, GE Healthcare, USA) on the MR operating console was applied to process the wave information automatically, and an inversion algorithm was used to generate the wave images, elastograms, and magnitude images with the crosshatched areas (low confidence data were excluded by the post-processing algorithm) $(28,29)$. Tissue stiffness value, which was measured in units of kilopascals $(\mathrm{kPa})$, could be measured on the elastogram. The primary physical measurements used in our work were the shear stiffness as $\mu$, with a physical unit of kilopascal of $\mathrm{kPa}$. The shear stillness $\mu$ was also defined as the effective modulus and was derived from the complex modulus $\mu_{\mathrm{r}}+\mathrm{i} \mu_{\mathrm{i}}\left(\mu_{\mathrm{r}}\right.$ is the storage modulus, and $\mu_{i}$ is the loss modulus reflecting the attenuation of a viscoelastic medium). At a particular frequency, the shear stiffness can be calculated using the equation: $\mu=\rho V_{s}^{2}$, where $\rho$ is the density of the material (typically assumed to be approximately $1,000 \mathrm{~kg} / \mathrm{m}^{3}$ ) and $\mathrm{V}_{\mathrm{s}}$ is the wave speed of the shear wave, a product of the operating frequency and the spatial wavelength (30). 
Table 1 MRE sequence parameters

\begin{tabular}{lc}
\hline Sequence & $\begin{array}{c}\text { 2D spin echo-echo planar } \\
\text { imaging (SE-EPI) }\end{array}$ \\
\hline Plane & Axis \\
Repetition time (ms) & 1,000 \\
Echo time (ms) & min full \\
Matrix & $64 \times 64$ \\
Field of view (cm) & $42 \times 42$ \\
Slice thickness/intersection & $10 / 5$ \\
gap (mm) & 7 \\
Number of slices & 3 \\
Nex & 250 \\
Band width (kHz) & 70 \\
Acquisition time (s) & \\
Frequency of driver (Hz) & 51 (split in three breath-holds) \\
Amplitude (\%) & 60 \\
Axis of motion encoding & 70 \\
gradients pulse & \\
\hline
\end{tabular}

MRE, magnetic resonance elastography; SE-EPI, spin echoecho planar imaging.

Liver and spleen stiffness were measured by two radiologists who had 10 months of experience in postprocessing MRE images and were blinded to the patients' clinical and pathological information. The radiologists measured the liver and spleen stiffness by manually drawing the regions of interest (ROIs) on every elastogram. The ROIs were acceptable when they met the following criteria: (I) each ROI covered the liver or spleen as largely as possible in the axial slices; (II) the area was $\geq 1.5 \mathrm{~cm}^{2}$; and (III) liver or spleen edge, large vessels, bile ducts, signal loss, blurring area, and crosshatching marks were avoided. Finally, the averages of the liver and spleen stiffness were measured by two radiologists and computed. In this study, ROIs were drawn on all 7 slices. The mean \pm SD and minimal and maximal ROI areas of the liver were $51.32 \pm 24.88 \mathrm{~cm}^{2}(59 \%$ of the entire visible liver area \pm 0.14 ), $15.37 \mathrm{~cm}^{2}$ (39\% of the entire visible liver area), and $99.59 \mathrm{~cm}^{2}$ (99\% of the entire visible liver area), respectively. The mean $\pm \mathrm{SD}$ and minimal and maximal ROI areas of the spleen were $23.08 \pm 10.99 \mathrm{~cm}^{2}$ (58\% of the entire visible spleen area \pm 0.10$), 5.27 \mathrm{~cm}^{2}$ ( $40 \%$ of the entire visible spleen area), and $47.02 \mathrm{~cm}^{2}$ (78\% of the entire visible spleen area), respectively.

\section{Serum fibrosis markers}

The values of serum biochemical markers were calculated as follows: APRI $=[$ AST $(\mathrm{U} / \mathrm{L}) /$ upper normal limit $]$ $\times 100 /$ platelets $\left(10^{9} / \mathrm{L}\right)$ and FIB-4 $=$ age $($ years $) \times$ AST $(\mathrm{U} / \mathrm{L}) /$ [platelets $\left(10^{9} / \mathrm{L}\right) \times$ alanine aminotransferase $\left.(\operatorname{ALT})^{1 / 2}(\mathrm{U} / \mathrm{L})\right]$.

\section{Statistical analysis}

Statistical analyses were performed with the SPSS 20.0 software (SPSS Inc., Chicago, IL, USA) and MedCalc 18.2 (MedCalc Software, Mariakerke, Belgium). The intraclass correlation coefficient (ICC) was used to assess interobserver agreement. Reliability was considered to be excellent when the ICC was greater than 0.8 , good when it was $0.6-0.8$, average when it was $0.4-0.6$ and poor when it was less than 0.4 (31). All the data were checked for normal distribution using the Shapiro-Wilk normality test. Continuous parameters were expressed as the mean $\pm \mathrm{SD}$ (normally distributed data) or median and 25\% and $75 \%$ interquartile ranges (non-normal distribution parameter). The differences in liver and spleen stiffness and serum markers among different fibrosis stages or inflammation grades were analyzed by one-way ANOVA or the KruskalWallis test. Spearman's rank correlation analysis was used to evaluate the correlations between the two parameters (e.g., liver stiffness, spleen stiffness, serum markers, and fibrosis stage). The correlations were defined as either very strong, moderately strong, fair, or weak if the absolute value of the correlation coefficient $(r)$ was at least $0.8,0.6-0.8,0.3-0.6$, less than 0.3 , respectively (32). Univariate linear regression analysis was performed to assess the relationships between inflammation activity in the liver and spleen stiffness measured by MRE.

Receiver operating characteristic (ROC) analysis was carried out to assess the performance of liver and spleen stiffness, serum markers (APRI and FIB-4), MRI parameters combined model (combined model 1), and the all-parameters combined model (combined model 2). The highest Youden index value was used as the criteria to obtain the cutoff points of the different diagnostic models with the best sensitivity and specificity. Areas under the curve (AUCs) with $95 \%$ confidence intervals, sensitivity, and specificity were used to identify the differences in $\mathrm{F} 0$ vs. F1-4, F0-1 vs. F2-4, F0-2 vs. F3-4, and $\mathrm{F} 0-3$ vs. F4. Multivariate logistic analysis was used to build combined models: combined model 1 contained the MR measurements for liver and spleen stiffness; and 
Table 2 Patient characteristics

\begin{tabular}{|c|c|}
\hline Parameters & Characteristics $(n=120)$ \\
\hline Age (years), mean \pm SD & $50 \pm 13$ \\
\hline Sex (male/female) & $33 / 87$ \\
\hline BMI $\left(\mathrm{kg} / \mathrm{m}^{2}\right)$, mean $\pm \mathrm{SD}$ & $23.11 \pm 2.85$ \\
\hline \multicolumn{2}{|l|}{ Fibrosis stage } \\
\hline Fo & 23 \\
\hline $\mathrm{F} 1$ & 22 \\
\hline $\mathrm{F} 2$ & 24 \\
\hline F3 & 23 \\
\hline $\mathrm{F} 4$ & 28 \\
\hline \multicolumn{2}{|l|}{ Inflammation grade } \\
\hline G0 & 24 \\
\hline G1 & 26 \\
\hline G2 & 38 \\
\hline G3 & 18 \\
\hline G4 & 14 \\
\hline \multicolumn{2}{|l|}{ Etiology } \\
\hline Hepatitis B infection & 39 \\
\hline Hepatitis C infection & 6 \\
\hline NASH/NAFLD & 33 \\
\hline Autoimmune & 9 \\
\hline Drug induced & 16 \\
\hline Alcohol induced & 11 \\
\hline Cryptogenic & 6 \\
\hline AST (U/L), median (Q1, Q3) & $50.50(26.85,86.35)$ \\
\hline ALT (U/L), median (Q1, Q3) & $55.00(29.50,115.00)$ \\
\hline Platelet count $\left(10^{9} / \mathrm{L}\right)$, mean $\pm \mathrm{SD}$ & $176.50 \pm 81.78$ \\
\hline FIB-4, median (Q1, Q3) & $2.05(1.15,3.80)$ \\
\hline APRI, median (Q1, Q3) & $0.71(0.39,1.43)$ \\
\hline
\end{tabular}

BMI, body mass index; AST, aspartate aminotransferase; ALT, aspartate aminotransferase; NASH/NAFLD, nonalcoholic steatohepatitis/nonalcoholic fatty liver disease; FIB-4, fibrosis index based on the 4 factors; APRI, AST to platelet ratio index; $\mathrm{SD}$, standard deviation; Q1, $25^{\text {th }}$ percentile; Q3, $75^{\text {th }}$ percentile.

combined model 2 contained all measurements for liver and spleen stiffness, APRI, and FIB-4. The AUC was used to evaluate the diagnostic performance of all models, and the differences were compared by the DeLong test. AUC values were interpreted as follows: excellent was higher than 0.90 ; good was $0.80-0.90$; fair was $0.70-0.80$; poor was $0.60-0.70$; failed was $0.50-0.60$ (33). For all analyses, a two-tailed $\mathrm{P}$ value of $<0.05$ was statistically significant.

\section{Results}

\section{Clinical features}

One hundred and twenty patients were included in this study. All demographics, clinical features, laboratory tests, and pathological results are shown in Table 2. Based on pathologic findings, 23 patients had no fibrosis, 22 patients had F1 stage, 24 patients had F2 stage, 23, and 28 patients were graded into $\mathrm{F} 3$ and $\mathrm{F} 4$ stage. The mean $\pm \mathrm{SD}$ or median ( $25 \%$ and $75 \%$ interquartile ranges) of liver and spleen stiffness, APRI, and FIB-4 for the different fibrosis stages are summarized in Table 3. Liver and spleen stiffness, APRI, and FIB-4 showed statistically significant differences among the five fibrosis stages (all $\mathrm{P}<0.001)$. Box plots of liver and spleen stiffness values from the MRE, FIB4, and APRI for fibrosis stage groups F0, F1, F2, F3, and F4, are shown in Figure 2. An example of study patients is illustrated in Figure 3.

\section{Interobserver consistency}

The ICCs for consistency between the two radiologists were excellent, with ICC agreements of 0.88 and 0.89 for liver and spleen stiffness, respectively $(\mathrm{P}<0.001)$.

\section{Correlation of noninvasive parameters with liver fibrosis stage}

The liver stiffness values determined by MRE had a very strong correlation with their pathology grade $(r=0.87$, $\mathrm{P}<0.001)$. There were moderately strong correlations between fibrosis stage and spleen stiffness, fibrosis stage and FIB-4, and fibrosis stage and ARPI $(r=0.64,0.65$, and 0.51 , respectively, all $\mathrm{P}<0.001$ ).

\section{Comparison of the diagnostic performance of MRI parameters and serum markers}

All of the AUCs, optimal cutoff values, and the sensitivity and specificity of the different diagnostic models for detection of any fibrosis (F0 vs. F1-4), significant fibrosis (F0-1 vs. F2-4), advanced fibrosis (F0-2 vs. F3-4), and 
Table 3 Comparisons of noninvasive parameters (liver stiffness, spleen stiffness, APRI and FIB-4) among different liver fibrosis stages (F0-4) and liver inflammation grades (G0-4)

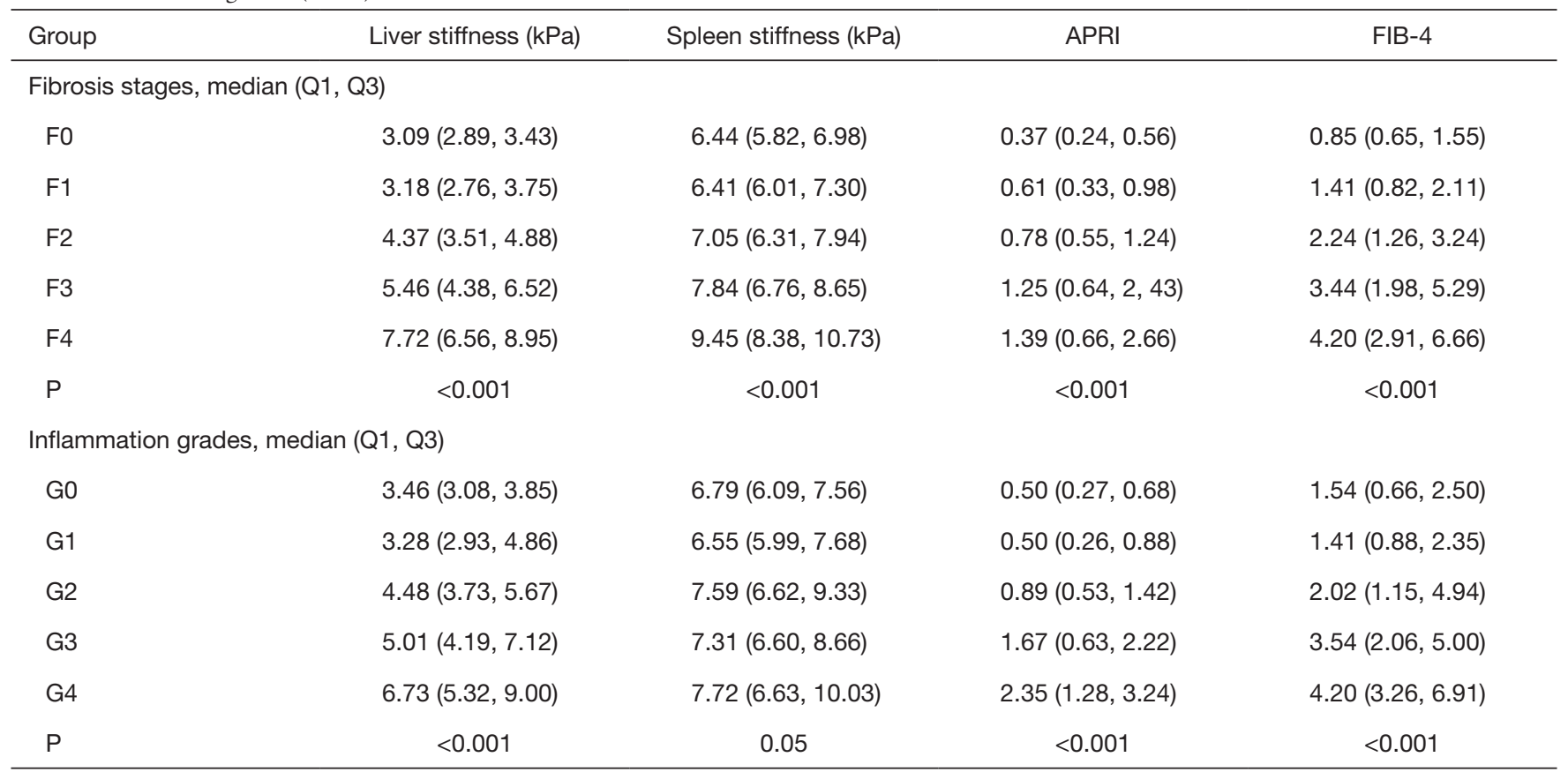

FIB-4, fibrosis index based on the 4 factors; APRI, aspartate aminotransferase-to-platelet ratio index; kPa, kilopascal; $\mathrm{Q} 1,25^{\text {th }}$ percentile; Q3, $75^{\text {th }}$ percentile.

cirrhosis (F0-3 vs. F4) are presented in Tables 4-7 and Figure 4. The AUC values of the liver stiffness and combined model 1 were significantly higher than those of APRI and FIB-4 in staging F2-4, F3-4, and F4 (all $\mathrm{P}<0.05)$. No significant differences were found among these 4 models in staging F1-4 ( $>0.05)$. In addition, FIB-4 had higher performance than APRI in staging F2-4, F3-4, and $\mathrm{F} 4(\mathrm{P}=0.04,0.02$, and 0.002 , respectively). The diagnostic value of combined model 1 was significantly higher than spleen stiffness in staging F1-4, F2-4, F3-4, and F4 ( $\mathrm{P}=0.02$, $<0.001,0.001$, and 0.01 , respectively). There were no significant differences among combined models 1 and 2 and liver stiffness in the fibrosis staging groups $(\mathrm{P}>0.05)$. Interestingly, when discriminating liver fibrosis from the low stage to the high stage, the AUC value of spleen stiffness value increased from 0.76 to 0.88 ; in contrast, the AUC value of APRI decreased from 0.82 to 0.70 .

\section{Influence of inflammation grade on liver and spleen stiffness}

Liver stiffness was not equal among different inflammation grades $(\mathrm{P}<0.001)$. Meanwhile, spleen stiffness showed no differences among the different inflammation grades $(\mathrm{P}=0.05)$. Details are shown in Table 3. Univariate linear regression analysis showed a positive correlation between inflammation activity and liver stiffness (adjusted $\mathrm{R}$ square $=0.22, \mathrm{P}<0.001)$, but inflammation was not statistically relevant to spleen stiffness $(\mathrm{P}=0.06)$.

\section{Discussion}

Our study aimed to stage liver fibrosis in a convenient and precise way. Therefore, we emphasized liver and spleen elastography as well as common serum markers in an attempt to discover a diagnostic model for staging liver fibrosis with high performance. This study revealed the diagnostic efficacy of liver and spleen stiffness measured by MRE, APRI, FIB-4, and MR measurements model (liver and spleen stiffness, combined model 1), as well as the allparameters model (all 4 measurements, combined model 2) for staging hepatic fibrosis. Our results validated that liver stiffness can grade hepatic fibrosis more accurately than any other single-measurement method. The diagnostic efficiency of combined model 2 was slightly elevated in staging mild fibrosis compared with that of liver stiffness, 

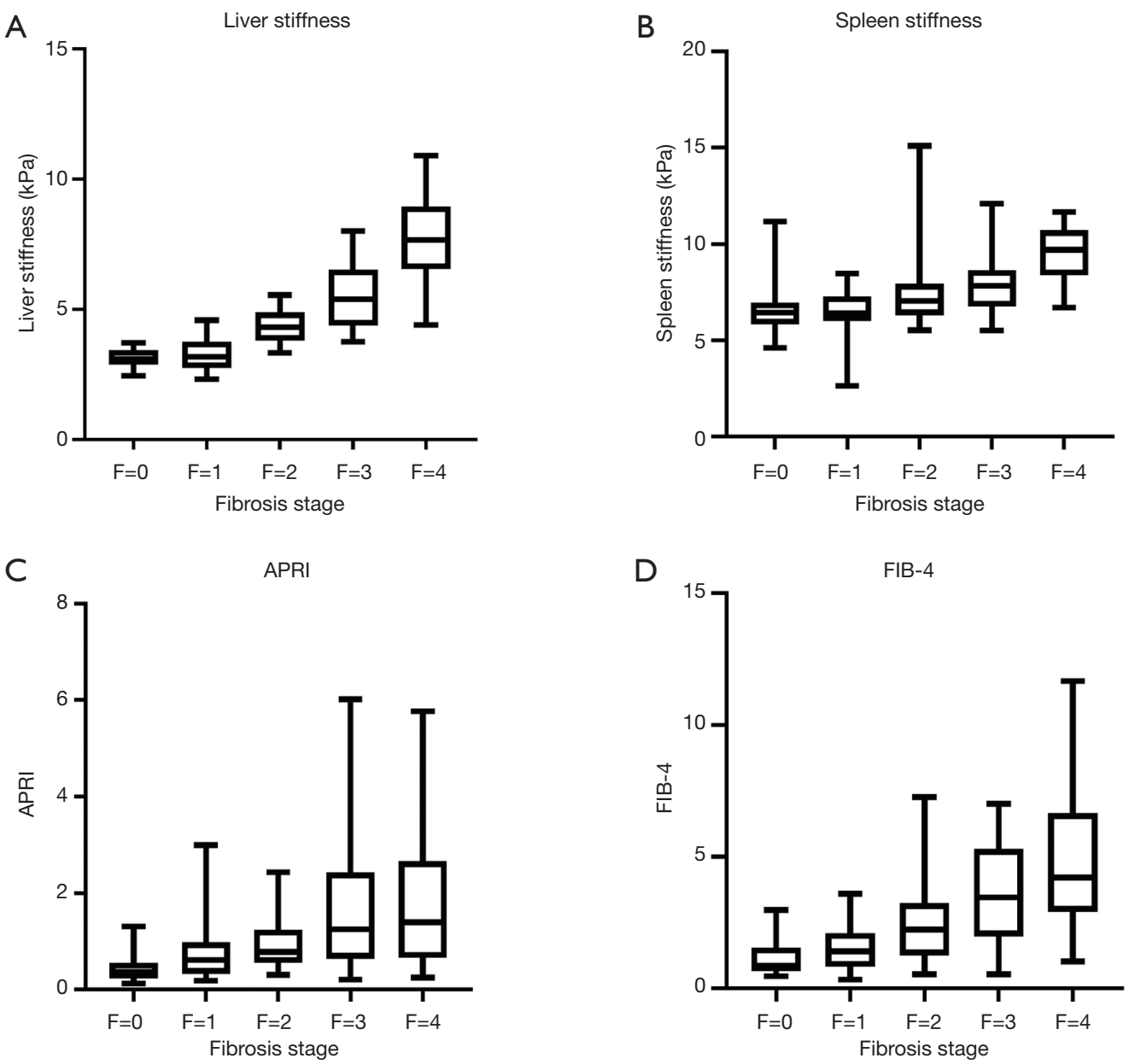

Figure 2 Box plots of liver and spleen stiffness measured by MRE, APRI, and FIB-4 for fibrosis stage groups F0, F1, F2, F3, and F4. All the parameters showed significant differences among the five fibrosis stages (all $\mathrm{P}<0.001)$. MRE, magnetic resonance elastography, APRI, aspartate aminotransferase-to-platelet ratio index; FIB-4, fibrosis index based on the 4 factors.

but the difference was not significant. The diagnostic performances of combined model 1 and combined model 2 were similar. Spleen stiffness showed higher performance and sensitivity in staging severe fibrosis than in staging no/ mild fibrosis. Even though all the parameters correlated with pathology grade, liver stiffness had the highest correlation among them.

Recent studies reported that liver stiffness had an excellent diagnostic performance. The AUCs for staging $\mathrm{F} 1-4, \mathrm{~F} 2-4, \mathrm{~F} 3-4$, and F4 were in the range of 0.82 $0.99,0.88-0.98,0.93-1.00$, and $0.92-1.00$, respectively. Additionally, liver stiffness also had a strong correlation with fibrosis stage (13,34-38). In our study, the AUCs for staging liver stiffness were consistent with those reported in previous studies. Liver stiffness showed higher diagnostic efficacy for staging patients with advanced fibrosis and cirrhosis than for staging patients with no fibrosis. The deposition of collagen fibers is the key point in hepatic fibrosis, which has considerable effects on the mechanical properties of the liver tissues. Following our results and previous studies, liver MRE can reflect this pathological process; however, in our study, the cutoff of F1-4 was higher than those in previous studies, and the cutoffs for stages F1-4 and F2-4 were similar (38). Several possible explanations for this could be as follows; first, different kinds of MR equipment, pulse sequences, and ROIs were used. Previous studies showed that the liver stiffness measured by MRE varies between different fields, sequences, and different selection methods of ROIs $(29,38,39)$. However, in this study, unified scan equipment 

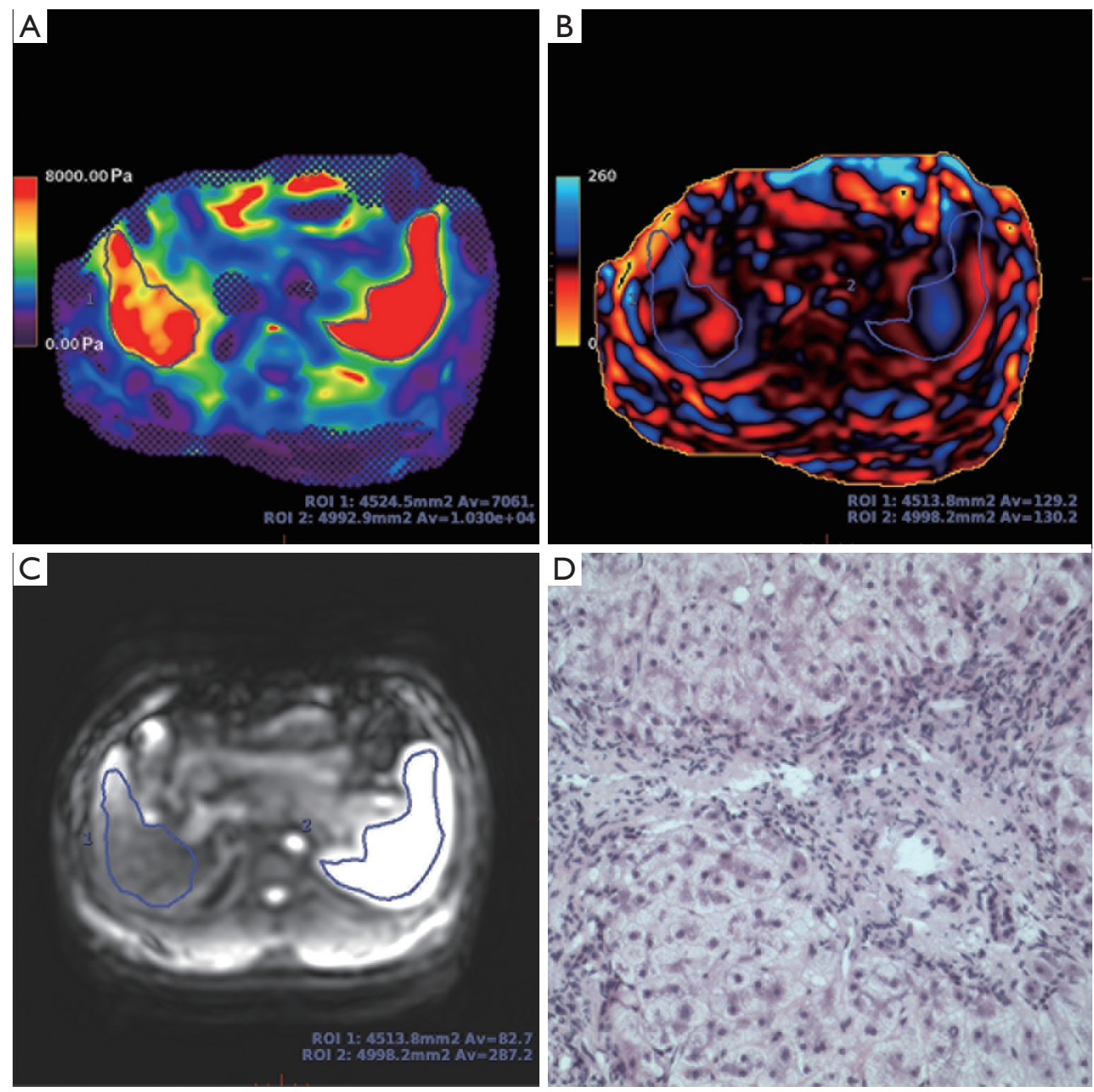

Figure 3 Images of a 42-year-old man with chronic hepatitis B. Elastogram (A) and wave image (B) were post-processed with software "Volume Viewer" (version 13.0, GE Healthcare, USA) on the magnetic resonance operating console after MRE acquisition. Liver stiffness is high and heterogeneous. Crosshatched areas representing low confidence data were excluded by the post-processing algorithm. The average liver and spleen stiffness were 7.57 and $8.49 \mathrm{kPa}$, respectively. (C) The magnitude image. (D) Liver biopsy specimen (HE staining, $\times 400$ ). The fibrous proliferation of interlobular portal areas could be found. The pathological fibrosis stage is F4. MRE, magnetic resonance elastography.

(3.0 Tesla field strength MR) and EPI sequence with lower failure rates (40) were used. For analysis, one or several, freehand or circular ROIs on the right liver lobe or different liver segments were drawn $(38,41)$. Since liver fibrosis is regarded as diffused liver disease, the larger ROIs were sketched to cover more liver tissue, providing more reliable stiffness values. Furthermore, we also noticed that Yoshimitsu et al. used the same MRI equipment and pulse sequence that we did to analyze 70 patients without acute hepatitis, and the cutoff for liver stiffness in identifying F0 vs. F1-4 (3.13 kPa) was still lower than in our study (12).
Thus, the second explanation for these differences was the type of patients we enrolled. The number of patients at different fibrosis stages was limited in our study, and many patients also had inflammation. From our statistical analysis, we found a positive correlation between inflammation activity and liver stiffness measured by MRE. This finding was consistent with previous studies (34). Inflammation is common in patients with fibrosis. Inflammatory infiltration can increase the blood supply and intrinsic stress, causing the hepatocytes to swell. These changes may have increased liver stiffness. Also, some studies have produced conflicting 
Table 4 Diagnostic performance of liver stiffness, spleen stiffness, APRI, FIB-4, combined model 1 (live and spleen stiffness combined model) and combined model 2 (all the four parameters combined model) for detection of any fibrosis (F0 vs. F1-4)

\begin{tabular}{|c|c|c|c|c|c|}
\hline Parameters & \multicolumn{5}{|c|}{ F0 vs. F1-4 (any fibrosis) } \\
\hline Liver stiffness $(\mathrm{kPa})$ & $>3.71$ & $0.89(0.82-0.94)$ & 78.35 & 100 & $<0.001$ \\
\hline Spleen stiffness (kPa) & $>6.62$ & $0.76(0.68-0.83)$ & 72.16 & 73.91 & $<0.001$ \\
\hline APRI & $>0.77$ & $0.82(0.74-0.88)$ & 56.70 & 95.65 & $<0.001$ \\
\hline Combined model $1^{\dagger}$ & $>0.79$ & $0.89(0.82-0.94)$ & 78.35 & 100 & $<0.001$ \\
\hline Combined model $2^{\ddagger}$ & $>0.83$ & $0.90(0.84-0.95)$ & 77.32 & 95.65 & $<0.001$ \\
\hline
\end{tabular}

${ }^{\dagger}$, combined model 1 represents liver and spleen stiffness combined model; ${ }^{\ddagger}$, combined model 2 represents liver stiffness, spleen stiffness, APRI, FIB-4 combined model. FIB-4, fibrosis index based on the 4 factors; APRI, AST to platelet ratio index; kPa, kilopascal; AUC, area under the curve; $\mathrm{Cl}$, confidence interval.

Table 5 Diagnostic performance of liver stiffness, spleen stiffness, APRI, FIB-4, combined model 1 (live and spleen stiffness combined model) and combined model 2 (all the four parameters combined model) for detection of significant fibrosis (F0-1 vs. F2-4)

\begin{tabular}{|c|c|c|c|c|c|}
\hline Parameters & \multicolumn{5}{|c|}{ F0-1 vs. F2-4 (significant fibrosis) } \\
\hline Liver stiffness (kPa) & $>3.71$ & $0.97(0.92-0.99)$ & 94.67 & 88.89 & $<0.001$ \\
\hline Spleen stiffness (kPa) & $>6.62$ & $0.82(0.73-0.88)$ & 82.67 & 68.89 & $<0.001$ \\
\hline APRI & $>0.72$ & $0.79(0.70-0.86)$ & 65.33 & 77.78 & $<0.001$ \\
\hline Combined model $1^{\dagger}$ & $>0.54$ & $0.97(0.92-0.99)$ & 89.33 & 93.33 & $<0.001$ \\
\hline Combined model $2^{\ddagger}$ & $>0.51$ & $0.97(0.92-0.99)$ & 92.00 & 91.11 & $<0.001$ \\
\hline
\end{tabular}

${ }^{\dagger}$, combined model 1 represents liver and spleen stiffness combined model; ${ }^{\ddagger}$, combined model 2 represents liver stiffness, spleen stiffness, APRI, FIB-4 combined model. FIB-4, fibrosis index based on the 4 factors; APRI, AST to platelet ratio index; kPa, kilopascal; AUC, area under the curve; $\mathrm{Cl}$, confidence interval.

Table 6 Diagnostic performance of liver stiffness, spleen stiffness, APRI, FIB-4, combined model 1 (live and spleen stiffness combined model) and combined model 2 (all the four parameters combined model) for detection of advanced fibrosis (F0-2 vs. F3-4)

\begin{tabular}{|c|c|c|c|c|c|}
\hline Parameters & \multicolumn{5}{|c|}{ F0-2 vs. F3-4 (advanced fibrosis) } \\
\hline Liver stiffness $(\mathrm{kPa})$ & $>4.74$ & $0.95(0.89-0.98)$ & 84.31 & 91.30 & $<0.001$ \\
\hline Spleen stiffness (kPa) & $>7.22$ & $0.83(0.75-0.89)$ & 86.27 & 75.36 & $<0.001$ \\
\hline APRI & $>1.1$ & $0.75(0.67-0.83)$ & 60.78 & 84.06 & $<0.001$ \\
\hline Combined model $1^{\dagger}$ & $>0.69$ & $0.95(0.89-0.98)$ & 76.47 & 98.55 & $<0.001$ \\
\hline Combined model $2^{\ddagger}$ & $>0.46$ & $0.95(0.89-0.98)$ & 84.31 & 91.30 & $<0.001$ \\
\hline
\end{tabular}

${ }^{\dagger}$, combined model 1 represents liver and spleen stiffness combined model; ${ }^{\ddagger}$, combined model 2 represents liver stiffness, spleen stiffness, APRI, FIB-4 combined model. FIB-4, fibrosis index based on the 4 factors; APRI, AST to platelet ratio index; kPa, kilopascal; AUC, area under the curve; $\mathrm{Cl}$, confidence interval. 
Table 7 Diagnostic performance of liver stiffness, spleen stiffness, APRI, FIB-4, combined model 1 (live and spleen stiffness combined model) and combined model 2 (all the four parameters combined model) for detection of cirrhosis (F0-3 vs. F4)

\begin{tabular}{|c|c|c|c|c|c|}
\hline Parameters & \multicolumn{5}{|c|}{ F0-3 vs. F4 (cirrhosis) } \\
\hline Liver stiffness (kPa) & $>6.04$ & $0.95(0.90-0.98)$ & 85.71 & 92.39 & $<0.001$ \\
\hline Spleen stiffness (kPa) & $>7.39$ & $0.88(0.80-0.93)$ & 92.86 & 72.83 & $<0.001$ \\
\hline APRI & $>0.95$ & $0.70(0.61-0.78)$ & 67.86 & 69.57 & $<0.001$ \\
\hline Combined model $1^{\dagger}$ & $>0.12$ & $0.96(0.90-0.99)$ & 96.43 & 83.70 & $<0.001$ \\
\hline Combined model $2^{\ddagger}$ & $>0.08$ & 0.96 (0.90-0.99) & 96.43 & 82.61 & $<0.001$ \\
\hline
\end{tabular}

${ }^{\dagger}$, combined model 1 represents liver and spleen stiffness combined model; ${ }^{\ddagger}$, combined model 2 represents liver stiffness, spleen stiffness, APRI, FIB-4 combined model. FIB-4, fibrosis index based on the 4 factors; APRI, AST to platelet ratio index; kPa, kilopascal; AUC, area under the curve; $\mathrm{Cl}$, confidence interval.

results regarding the influence of inflammation on liver stiffness in chronic liver disease, which may be explained by variations in the inflammation grade (42). Third, the METAVIR score can be influenced by biopsy specimens. The pathological stage was based on a limited number of tissues, and the definitions of F0 and F1 may be unclear (25). However, liver MRE can reflect the fibrosis of the whole heterogeneous liver. Besides, our results indicated that liver stiffness had better accuracy than any other single diagnostic model, and there were no statistical differences from the AUC tests between liver stiffness and the combined models in staging F1-4, F2-4, F3-4, and F4. Therefore, only liver stiffness is reliable and acceptable for diagnosing hepatic fibrosis in clinic.

Few studies have focused on the diagnostic power of spleen stiffness measured by ultrasound or MRE in the precise staging of liver fibrosis. Our study showed that spleen stiffness had a moderately strong correlation with fibrosis stage, which was slightly higher than that reported in previous literature (43). Furthermore, the diagnostic performance of spleen stiffness increased with increased hepatic fibrosis. Spleen stiffness showed higher sensitivity in staging advanced fibrosis and cirrhosis than in staging mild fibrosis. Pathophysiological changes in chronic liver disease resulted in spleen stiffness variance. These changes were exemplified in the increase of spleen blood flow and hyperplasia of spleen tissue, especially for advanced fibrosis. Several studies have verified that spleen stiffness is correlated with liver stiffness and hepatic venous pressure gradient $(22,44,45)$. Also, inflammation activity was not a confounding factor of spleen stiffness; therefore, spleen stiffness could help stage liver fibrosis in patients with inflammation. However, in our study, the AUCs of spleen stiffness were lower than those of liver stiffness in staging fibrosis. Also, combined model 1 did not significantly elevate the AUC compared with liver stiffness. Notably, Cheng et al. found a similar AUC of spleen stiffness in diagnosing advanced fibrosis with the passive driver located over the spleen, and their studies also showed no significant differences with using the combined model and liver stiffness (43). Hu et al. used FibroScan and found that the diagnostic performance of spleen stiffness was comparable with that of liver stiffness (46). These differences may be attributed to unrepresentative patient samples and different equipment.

Nonetheless, Reiter et al. recently used tomoelastography by multifrequency MRE of the liver and spleen and found that the combined analysis of liver and spleen had higher diagnostic performance than liver stiffness alone in F4 patients (47). Except for the differences in systemic pathology, the technical innovation of MRE may affect the evaluation in the diagnostic performance of liver and spleen stiffness. Further studies have focused on acquiring spleen stiffness quickly and reliably are needed.

APRI and FIB-4 are common and easily available serum biomarkers recommended by the World Health Organization for the identification of liver fibrosis in patients with chronic liver disease. We found that FIB-4 and APRI had fair accuracy as well as moderate sensitivity and specificity for detecting different fibrosis stages. The AUC values of FIB-4 were significantly higher than those of APRI in grading F2-4, F3-4, and F4, which was consistent 

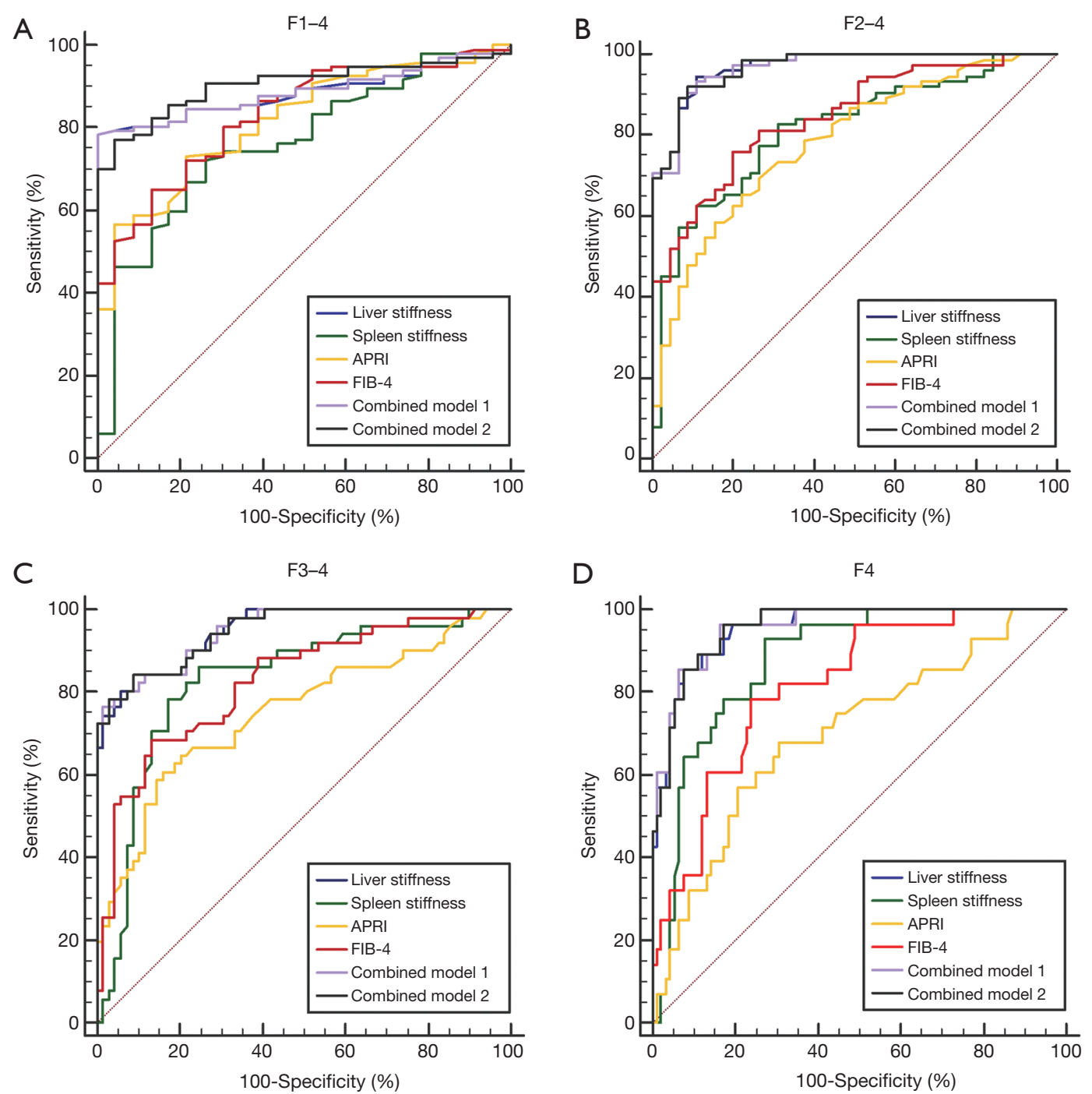

Figure 4 Receiver operating characteristic curves of liver stiffness, spleen stiffness, APRI, FIB-4, combined model 1 (liver and spleen stiffness combined model), and combined model 2 (liver and spleen stiffness, APRI, and FIB-4 combined model) for detecting any fibrosis (F1-4), significant fibrosis (F2-4), advanced fibrosis (F3-4), cirrhosis (F4). APRI, aspartate aminotransferase-to-platelet ratio index; FIB-4, fibrosis index based on the 4 factors.

with previous studies $(17,48,49)$. Nevertheless, a recent meta-analysis found no significant differences between FIB-4 and APRI in diagnosing significant fibrosis (50). With the assessment of FIB-4 involving age and ALT, age was reported to be associated with cirrhosis. Some studies have demonstrated that AST was more elevated than ALT as fibrosis became increasingly aggravated, leading to delayed clearance of AST and mitochondrial injury in more advanced-stage fibrosis (48). Also, the AUC value of APRI decreased with increasing fibrosis grade, and the previous study did not show this trend (17). AST and platelet count can be influenced by extrahepatic lesions (9); APRI alone was not reliable enough to precisely stage fibrosis.

We separately tested two combined diagnostic models to compare single-measurement and combined diagnostic models. The two models were the liver and spleen stiffness combined model (combined model 1) and all obtained parameters combined in one (combined model 2). Combined model 1 was superior to biochemistry-alone diagnosis and also had higher sensitivity and specificity, especially in staging F2-4, F3-4, and F4. Extrahepatic diseases or other hepatic pathologic changes can affect 
serum markers (9); therefore, imaging examinations may be more advantageous. Besides, both combined models mostly showed similar diagnostic value. Only in the prediction of F1-4 did the combined model 2 have a slightly higher AUC value than combined model 1; although, the difference was not significant. Moreover, no significant difference was found between the combined models and liver stiffness. Hence, liver stiffness is not only convenient but also dependable for staging hepatic fibrosis in clinic. For early fibrosis diagnosis, combined model 2 may be more useful than other diagnostic models.

Liver fibrosis is a dynamic process that can be controlled by timely treatment. Robust and convenient noninvasive biomarkers are pivotal for guiding therapy choice and evaluating treatment effects.

Our study has clinical implications; MR-based techniques can reflect not only anatomical changes but also fibrosis stage and complications. Further comprehensive studies about liver and spleen MRE, as well as other promising imaging markers, are needed in the future.

Our study has some potential limitations. First, this was a retrospective study, and selection bias might exist, causing an unbalanced number of patients in different fibrosis stages. Second, patients had pooled etiologies. The pathological process varied among the distinct types; therefore, evaluating the biomarkers may be biased. Moreover, patients at the same fibrosis stage had various pathological degrees of inflammation and steatosis, which also affected stiffness. The influences patients with different etiologies had on the results are also controversial (38). Restricted etiology and confounding factors of stiffness should be taken into consideration. Third, although some studies mentioned that liver and spleen stiffness could be obtained simultaneously just like our study $(51,52)$, studies with a limited number of patients, such as the studies by Mannelli et al. and Cheng et al., reported that there were differences in spleen stiffness acquired in the different locations of the passive driver. They recommend that the passive driver should be placed on the left chest wall so that the excitation wave can traverse a longer length of the spleen and generate larger wave amplitudes for the following analysis $(43,53,54)$. Therefore, further studies comparing the different driver locations in the same patients or tomoelastography of the liver and spleen need to be explored as they may be helpful to improve the confidence level of spleen stiffness $(47,53)$.

In conclusion, liver stiffness measured with MRE had better performance than spleen stiffness, APRI, and FIB4 for staging liver fibrosis. The combined models did not significantly improve diagnostic value compared with liver stiffness in staging fibrosis. Furthermore, spleen stiffness may be feasible for the detection of cirrhosis, and this needs to be studied further.

\section{Acknowledgments}

Funding: This work was supported by the National Natural Science Foundation of China (No. 61871276), Capital's Funds for Health Improvement and Research (No. 20182-2023), and Beijing Natural Science Foundation (No. 7184199).

\section{Footnote}

Conflicts of Interest: All authors have completed the ICMJE uniform disclosure form (available at http://dx.doi. org/10.21037/qims-19-849). The authors have no conflicts of interest to declare.

Ethical Statement: This study was approved by our institutional review board. Written informed consent was obtained from all patients.

Open Access Statement: This is an Open Access article distributed in accordance with the Creative Commons Attribution-NonCommercial-NoDerivs 4.0 International License (CC BY-NC-ND 4.0), which permits the noncommercial replication and distribution of the article with the strict proviso that no changes or edits are made and the original work is properly cited (including links to both the formal publication through the relevant DOI and the license). See: https://creativecommons.org/licenses/by-nc-nd/4.0/.

\section{References}

1. GBD 2017 Mortality Collaborators. Global, regional, and national age-sex-specific mortality and life expectancy, 1950-2017: a systematic analysis for the Global Burden of Disease Study 2017. Lancet 2018;392:1684-735.

2. Asrani SK, Devarbhavi H, Eaton J, Kamath PS. Burden of liver diseases in the world. J Hepatol 2019;70:151-71.

3. Lee YA, Wallace MC, Friedman SL. Pathobiology of liver fibrosis: a translational success story. Gut 2015;64:830-41.

4. Lee UE, Friedman SL. Mechanisms of hepatic fibrogenesis. Best Pract Res Clin Gastroenterol 2011;25:195-206.

5. Rockey DC, Caldwell SH, Goodman ZD, Nelson RC, 
Smith AD. Liver biopsy. Hepatology 2009;49:1017-44.

6. Ratziu V, Charlotte F, Heurtier A, Gombert S, Giral P, Bruckert E, Grimaldi A, Capron F, Poynard T. Sampling variability of liver biopsy in nonalcoholic fatty liver disease. Gastroenterology 2005;128:1898-906.

7. Vallet-Pichard A, Mallet V, Nalpas B, Verkarre V, Nalpas A, Dhalluin-Venier V, Fontaine H, Pol S. FIB-4: an inexpensive and accurate marker of fibrosis in $\mathrm{HCV}$ infection. comparison with liver biopsy and fibrotest. Hepatology 2007;46:32-36.

8. Wai CT, Greenson JK, Fontana RJ, Kalbfleisch JD, Marrero JA, Conjeevaram HS, Lok AS. A simple noninvasive index can predict both significant fibrosis and cirrhosis in patients with chronic hepatitis C. Hepatology 2003;38:518-26.

9. Castera L, Pinzani M. Biopsy and non-invasive methods for the diagnosis of liver fibrosis: does it take two to tango? Gut 2010;59:861-66.

10. Lurie Y, Webb M, Cytter-Kuint R, Shteingart S, Lederkremer GZ. Non-invasive diagnosis of liver fibrosis and cirrhosis. World J Gastroenterol 2015;21:11567-83.

11. Hines CD, Bley TA, Lindstrom MJ, Reeder SB. Repeatability of magnetic resonance elastography for quantification of hepatic stiffness. J Magn Reson Imaging 2010;31:725-31.

12. Yoshimitsu K, Mitsufuji T, Shinagawa Y, Fujimitsu R, Morita A, Urakawa H, Hayashi H, Takano K. MR elastography of the liver at $3.0 \mathrm{~T}$ in diagnosing liver fibrosis grades; preliminary clinical experience. Eur Radiol 2016;26:656-63.

13. Singh S, Venkatesh SK, Wang Z, Miller FH, Motosugi U, Low RN, Hassanein T, Asbach P, Godfrey EM, Yin M, Chen J, Keaveny AP, Bridges M, Bohte A, Murad MH, Lomas DJ, Talwalkar JA, Ehman RL. Diagnostic performance of magnetic resonance elastography in staging liver fibrosis: a systematic review and meta-analysis of individual participant data. Clin Gastroenterol Hepatol 2015;13:440-51.e6.

14. Wáng YX. Living tissue intravoxel incoherent motion (IVIM) diffusion MR analysis without $\mathrm{b}=0$ image: an example for liver fibrosis evaluation. Quant Imaging Med Surg 2019;9:127-33.

15. Xu J, Wang X, Jin ZY, You Y, Wang Q, Wang ST, Xue HD. Value of Texture Analysis on Gadoxetic Acidenhanced MR for Detecting Liver Fibrosis in a Rat Model. Chin Med Sci J 2019;34:24-32.

16. Hennedige TP, Wang G, Leung FP, Alsaif HS, Teo LL, Lim SG, Wee A, Venkatesh SK. Magnetic Resonance
Elastography and Diffusion Weighted Imaging in the Evaluation of Hepatic Fibrosis in Chronic Hepatitis B. Gut Liver 2017;11:401-8.

17. Besa C, Wagner M, Lo G, Gordic S, Chatterii M, Kennedy P, Stueck A, Thung S, Babb J, Smith A, Taouli B. Detection of liver fibrosis using qualitative and quantitative MR elastography compared to liver surface nodularity measurement, gadoxetic acid uptake, and serum markers. J Magn Reson Imaging 2018;47:1552-61.

18. Choi YR, Lee JM, Yoon JH, Han JK, Choi BI. Comparison of magnetic resonance elastography and gadoxetate disodium-enhanced magnetic resonance imaging for the evaluation of hepatic fibrosis. Invest Radiol 2013;48:607-13.

19. Ichikawa S, Motosugi U, Nakazawa T, Morisaka H, Sano K, Ichikawa T, Enomoto N, Matsuda M, Fujii H, Onishi $\mathrm{H}$. Hepatitis activity should be considered a confounder of liver stiffness measured with MR elastography. J Magn Reson Imaging 2015;41:1203-8.

20. Tang A, Cloutier G, Szeverenyi NM, Sirlin CB. Ultrasound Elastography and MR Elastography for Assessing Liver Fibrosis: Part 2, Diagnostic Performance, Confounders, and Future Directions. AJR Am J Roentgenol 2015;205:33-40.

21. Sun HY, Lee JM, Han JK, Choi BI. Usefulness of MR elastography for predicting esophageal varices in cirrhotic patients. J Magn Reson Imaging 2014;39:559-66.

22. Talwalkar JA, Yin M, Venkatesh S, Rossman PJ, Grimm RC, Manduca A, Romano A, Kamath PS, Ehman RL. Feasibility of in vivo MR elastographic splenic stiffness measurements in the assessment of portal hypertension. AJR Am J Roentgenol 2009;193:122-27.

23. Takuma Y, Morimoto Y, Takabatake H, Toshikuni N, Tomokuni J, Sahara A, Matsueda K, Yamamoto H. Measurement of Spleen Stiffness With Acoustic Radiation Force Impulse Imaging Predicts Mortality and Hepatic Decompensation in Patients With Liver Cirrhosis. Clin Gastroenterol Hepatol 2017;15:1782-90.e4.

24. Berzigotti A, Seijo S, Arena U, Abraldes JG, Vizzutti F, Garcia-Pagan JC, Pinzani M, Bosch J. Elastography, spleen size, and platelet count identify portal hypertension in patients with compensated cirrhosis. Gastroenterology 2013;144:102-11.e1.

25. Bedossa P, Poynard T. An algorithm for the grading of activity in chronic hepatitis C. The METAVIR Cooperative Study Group. Hepatology 1996;24:289-93.

26. Scheuer PJ. Classification of chronic viral hepatitis: a need for reassessment. J Hepatol 1991;13:372-74.

27. Yin M, Talwalkar JA, Glaser KJ, Manduca A, Grimm RC, 
Rossman PJ, Fidler JL, Ehman RL. Assessment of hepatic fibrosis with magnetic resonance elastography. Clin Gastroenterol Hepatol 2007;5:1207-13.e2.

28. Silva AM, Grimm RC, Glaser KJ, Fu Y, Wu T, Ehman RL, Silva AC. Magnetic resonance elastography: evaluation of new inversion algorithm and quantitative analysis method. Abdom Imaging 2015;40:810-17.

29. Trout AT, Serai S, Mahley AD, Wang H, Zhang Y, Zhang B, Dillman JR. Liver Stiffness Measurements with MR Elastography: Agreement and Repeatability across Imaging Systems, Field Strengths, and Pulse Sequences. Radiology 2016;281:793-804.

30. Mariappan YK, Glaser KJ, Ehman RL. Magnetic resonance elastography: a review. Clin Anat 2010;23:497-511.

31. Landis JR, Koch GG. The measurement of observer agreement for categorical data. Biometrics 1977;33:159-74.

32. Chan YH. Biostatistics 104: correlational analysis. Singapore Med J 2003;44:614-19.

33. Araújo MB, Pearson RG, Thuiller W, Erhard M. Validation of species-climate impact models under climate change. Global Change Biology 2005;11:1504-13.

34. Shi Y, Guo Q, Xia F, Dzyubak B, Glaser KJ, Li Q, Li J, Ehman RL. MR elastography for the assessment of hepatic fibrosis in patients with chronic hepatitis B infection: does histologic necroinflammation influence the measurement of hepatic stiffness? Radiology 2014;273:88-98.

35. Venkatesh SK, Wang G, Lim SG, Wee A. Magnetic resonance elastography for the detection and staging of liver fibrosis in chronic hepatitis B. Eur Radiol 2014;24:70-8.

36. Wang QB, Zhu H, Liu HL, Zhang B. Performance of magnetic resonance elastography and diffusion-weighted imaging for the staging of hepatic fibrosis: A meta-analysis. Hepatology 2012;56:239-47.

37. Singh S, Venkatesh SK, Loomba R, Wang Z, Sirlin C, Chen J, Yin M, Miller FH, Low RN, Hassanein T, Godfrey EM, Asbach P, Murad MH, Lomas DJ, Talwalkar JA, Ehman RL. Magnetic resonance elastography for staging liver fibrosis in non-alcoholic fatty liver disease: a diagnostic accuracy systematic review and individual participant data pooled analysis. Eur Radiol 2016;26:1431-40.

38. Garteiser P, Doblas S, Van Beers BE. Magnetic resonance elastography of liver and spleen: Methods and applications. Nmr Biomed 2018;31:e3891.

39. Bae JS, Lee JM, Park SJ, Lee KB, Han JK. Magnetic resonance elastography of healthy livers at 3.0 T: Normal liver stiffness measured by SE-EPI and GRE. Eur J Radiol 2018;107:46-53.

40. Zhan C, Kannengiesser S, Chandarana H, Fenchel M,
Ream J, Shanbhogue KP. MR elastography of liver at 3 Tesla: comparison of gradient-recalled echo (GRE) and spin-echo (SE) echo-planar imaging (EPI) sequences and agreement across stiffness measurements. Abdom Radiol (NY) 2019;44:1825-33.

41. Rusak G, Zawada E, Lemanowicz A, Serafin Z. Wholeorgan and segmental stiffness measured with liver magnetic resonance elastography in healthy adults: significance of the region of interest. Abdom Imaging 2015;40:776-82.

42. Leitão HS, Doblas S, Garteiser P, D'Assignies G, Paradis V, Mouri F, Geraldes CF, Ronot M, Van Beers BE. Hepatic Fibrosis, Inflammation, and Steatosis: Influence on the MR Viscoelastic and Diffusion Parameters in Patients with Chronic Liver Disease. Radiology 2017;283:98-107.

43. Cheng YW, Chang YC, Chen YL, Chen RC, Chou CT. Feasibility of measuring spleen stiffness with MR elastography and splenic volume to predict hepatic fibrosis stage. Plos One 2019;14:e0217876.

44. Nedredal GI, Yin M, McKenzie T, Lillegard J, LuebkeWheeler J, Talwalkar J, Ehman R, Nyberg SL. Portal hypertension correlates with splenic stiffness as measured with MR elastography. J Magn Reson Imaging 2011;34:79-87.

45. Ronot M, Lambert S, Elkrief L, Doblas S, Rautou PE, Castera L, Vilgrain V, Sinkus R, Van Beers BE, Garteiser P. Assessment of portal hypertension and highrisk oesophageal varices with liver and spleen threedimensional multifrequency MR elastography in liver cirrhosis. Eur Radiol 2014;24:1394-402.

46. Hu X, Xu X, Zhang Q, Zhang H, Liu J, Qian L. Indirect prediction of liver fibrosis by quantitative measurement of spleen stiffness using the FibroScan system. J Ultrasound Med 2014;33:73-81.

47. Reiter R, Tzschatzsch H, Schwahofer F, Haas M, Bayerl C, Muche M, Klatt D, Majumdar S, Uyanik M, Hamm B, Braun J, Sack I, Asbach P. Diagnostic performance of tomoelastography of the liver and spleen for staging hepatic fibrosis. Eur Radiol 2020;30:1719-29.

48. Xiao G, Yang J, Yan L. Comparison of diagnostic accuracy of aspartate aminotransferase to platelet ratio index and fibrosis-4 index for detecting liver fibrosis in adult patients with chronic hepatitis B virus infection: a systemic review and meta-analysis. Hepatology 2015;61:292-302.

49. Lu Q, Lu C, Li J, Ling W, Qi X, He D, Liu J, Wen T, Wu H, Zhu H, Luo Y. Stiffness Value and Serum Biomarkers in Liver Fibrosis Staging: Study in Large Surgical Specimens in Patients with Chronic Hepatitis B. Radiology 2016;280:290-9. 
50. Xu XY, Wang WS, Zhang QM, Li JL, Sun JB, Qin TT, Liu HB. Performance of common imaging techniques vs serum biomarkers in assessing fibrosis in patients with chronic hepatitis B: A systematic review and meta-analysis. World J Clin Cases 2019;7:2022-37.

51. Venkatesh SK, Ehman RL. Magnetic resonance elastography of abdomen. Abdom Imaging 2015;40:745-59.

52. Yoon H, Shin HJ, Kim MJ, Han SJ, Koh H, Kim S, Lee MJ. Predicting gastroesophageal varices through spleen magnetic resonance elastography in pediatric liver fibrosis.

Cite this article as: Wang XP, Wang Y, Ma H, Wang H, Yang DW, Zhao XY, Jin EH, Yang ZH. Assessment of liver fibrosis with liver and spleen magnetic resonance elastography, serum markers in chronic liver disease. Quant Imaging Med Surg 2020;10(6):1208-1222. doi: 10.21037/qims-19-849
World J Gastroenterol 2019;25:367-77.

53. Dyvorne HA, Jajamovich GH, Besa C, Cooper N, Taouli B. Simultaneous measurement of hepatic and splenic stiffness using MR elastography: preliminary experience. Abdom Imaging 2015;40:803-9.

54. Mannelli L, Godfrey E, Joubert I, Patterson AJ, Graves MJ, Gallagher FA, Lomas DJ. MR elastography: Spleen stiffness measurements in healthy volunteers--preliminary experience. AJR Am J Roentgenol 2010;195:387-92. 